\title{
THE HUBBLE SPACE TELESCOPE UV LEGACY SURVEY OF GALACTIC GLOBULAR CLUSTERS: THE INTERNAL KINEMATICS OF THE MULTIPLE STELLAR POPULATIONS IN NGC 2808*
}

\author{
A. Bellini ${ }^{1}$, E. Vesperini ${ }^{2}$, G. Piotto ${ }^{3,4}$, A. P. Milone ${ }^{5}$, J. Hong ${ }^{2}$, J. Anderson ${ }^{1}$, R. P. van Der Marel ${ }^{1}$, \\ L. R. Bedin ${ }^{4}$, S. Cassisi ${ }^{6,7}$, F. D’Antona ${ }^{8}$, A. F. Marino ${ }^{5}$, And A. Renzini ${ }^{4}$ \\ ${ }^{1}$ Space Telescope Science Institute, 3700 San Martin Drive, Baltimore, MD 21218, USA \\ ${ }^{2}$ Department of Astronomy, Indiana University, Bloomington, IN 47405, USA \\ 3 Dipartimento di Fisica e Astronomia "Galileo Galilei," Università di Padova, v.co dell'Osservatorio 3, I-35122, Padova, Italy \\ ${ }^{4}$ Istituto Nazionale di Astrofisica, Osservatorio Astronomico di Padova, v.co dell'Osservatorio 5, I-35122, Padova, Italy \\ ${ }^{5}$ Research School of Astronomy \& Astrophysics, Australian National University, Mt Stromlo Observatory, via Cotter Rd, Weston, ACT 2611, Australia \\ ${ }^{6}$ Istituto Nazionale di Astrofisica, Osservatorio Astronomico di Teramo, Via Mentore Maggini s.n.c., I-64100, Teramo, Italy \\ ${ }^{7}$ Instituto de Astrofisica de Canarias, E-38200 La Laguna, Tenerife, Canary Islands, Spain \\ ${ }^{8}$ Istituto Nazionale di Astrofisica, Osservatorio Astronomico di Roma, Via Frascati 33, I-00040, Monteporzio Catone, Roma, Italy \\ Received 2015 June 17; accepted 2015 August 6; published 2015 August 28
}

\begin{abstract}
Numerous observational studies have revealed the ubiquitous presence of multiple stellar populations in globular clusters and cast many difficult challenges for the study of the formation and dynamical history of these stellar systems. In this Letter we present the results of a study of the kinematic properties of multiple populations in NGC 2808 based on high-precision Hubble Space Telescope proper-motion measurements. In a recent study, Milone et al. identified five distinct populations (A-E) in NGC 2808. Populations D and E coincide with the heliumenhanced populations in the middle and the blue main sequences (mMS and bMS) previously discovered by Piotto et al.; populations A-C correspond to the redder main sequence that, in Piotto et al., was associated with the primordial stellar population. Our analysis shows that, in the outermost regions probed (between about 1.5 and 2 times the cluster half-light radius), the velocity distribution of populations $D$ and $E$ is radially anisotropic (the deviation from an isotropic distribution is significant at the $\sim 3.5 \sigma$ level). Stars of populations $\mathrm{D}$ and $\mathrm{E}$ have a smaller tangential velocity dispersion than those of populations $\mathrm{A}-\mathrm{C}$, while no significant differences are found in the radial velocity dispersion. We present the results of a numerical simulation showing that the observed differences between the kinematics of these stellar populations are consistent with the expected kinematic fingerprint of the diffusion toward the cluster outer regions of stellar populations initially more centrally concentrated.
\end{abstract}

Key words: Galaxy: kinematics and dynamics - globular clusters: individual (NGC 2808) - proper motions stars: Population III

\section{INTRODUCTION}

Photometric and spectroscopic studies over the last 15 years have revolutionized our understanding of globular clusters (GCs). Once thought to be "simple stellar populations" with a single age and composition, essentially all GCs host multiple stellar populations (MSPs), as revealed by their multiple photometric sequences. The exquisite precision and stability of the Hubble Space Telescope (HST) has made much of this possible (see Milone et al. 2015a, 2015b; Nardiello et al. 2015; Piotto et al. 2015, and references therein). MSPs can be traced to different compositions in terms of light elements (such as $\mathrm{Na}, \mathrm{O}, \mathrm{Al}$, and $\mathrm{Mg}$ observed spectroscopically; see Gratton et al. 2012 and references therein) and He (Pasquini et al. 2011; Villanova et al. 2012; Marino et al. 2014). These observational findings have cast a number of challenges for the study of the formation and evolution of GCs.

In order to make progress toward a complete picture of the formation and evolutionary history of GCs, as well as to constrain the possible paths for the theoretical study of these complex challenges and questions, a major effort to combine observational data is essential. Spectroscopic and photometric studies have begun to shed light on the chemical properties,

\footnotetext{
* Based on observations with the NASA/ESA Hubble Space Telescope, obtained at the Space Telescope Science Institute, which is operated by AURA, Inc., under NASA contract NAS 5-26555.
}

number of distinct sequences, and range of ages of different stellar populations.

Structural and kinematic properties of different stellar populations are two additional key pieces of the puzzle, as they contain essential information to build a complete picture of MSP cluster formation and dynamical evolution. According to a number of different formation scenarios (see, e.g., Decressin et al. 2007; D'Ercole et al. 2008; Bastian et al. 2013), second-generation (2G) populations should form more concentrated in the cluster inner regions. ${ }^{9}$ Numerical simulations (Vesperini et al. 2013) have shown that several clusters should still retain some memory of this initial spatial segregation and a few observational studies have indeed found clusters in which $2 \mathrm{G}$ stars are still concentrated in the cluster inner regions (Sollima et al. 2007; Bellini et al. 2009a, 2013b; Lardo et al. 2011; Johnson \& Pilachowski 2012; Milone et al. 2012; Cordero et al. 2014).

Little is however known to-date on the proper-motion (PM) based kinematics of MSPs (with the only exceptions of $\omega$ Cen and 47 Tuc: Bellini et al. 2009b; Anderson \& van der Marel 2010; Richer et al. 2013). Richer et al. (2013) probed the outer regions of 47 Tuc (at $\sim 1.9 r_{\mathrm{h}}$ ) and found that $2 \mathrm{G}$ stars are characterized by a radially anisotropic velocity distribution while the $1 \mathrm{G}$ population is isotropic. As discussed in Section 4,

\footnotetext{
9 Hereafter, we will use "populations" and "generations" as synonyms.
} 
this trend is consistent with the expected kinematical implications of the formation models mentioned above; the study presented in this Letter is aimed at exploring whether the kinematics of MSPs in NGC 2808 further confirms this general expectation and the trend identified in 47 Tuc.

NGC 2808 is one of the most massive Galactic GCs one of the few known to host a super-He-rich subpopulation (see, e.g., D'Antona et al. 2005; Piotto et al. 2007). The initial picture of three distinct stellar populations found by Piotto et al. (2007) has recently been shown to be even more complex by Milone et al. (2015b), who identified five distinct populations (named A-E) along the red-giant branch (RGB) and the main sequence (MS) of NGC 2808. We point out here that populations D and E correspond to the $2 \mathrm{G}$ populations identified by Piotto et al. (2007, their mMs and bMS). Populations A-C correspond to the red main sequence (rMS) of the Piotto et al. (2007) study (that they assumed to be the first generation, 1G). New WFC3/ UVIS UV data (GO-12605, PI: Piotto) allowed us to further split the rMS into the three populations, A-C. Available spectroscopic data discussed in Milone et al. (2015b) suggest that population $\mathrm{B}$ has $\mathrm{Na}$ and $\mathrm{O}$ abundances usually associated with $1 \mathrm{G}$ stars; population $\mathrm{C}$ includes stars with slightly enhanced $\mathrm{Na}$ and slightly depleted $\mathrm{O}$ abundances, and is photometrically distinct from population B along the RGB (see Milone et al. 2015b, their Figure 8). No spectroscopic data are available to further characterize population A.

In this Letter we shed further light on the properties of this complex cluster and present an analysis of the internal PMs of its five stellar populations. The outline of this Letter is as follows. In Section 2 we describe the data sets and reduction; in Section 3 we present our results on the kinematic properties of the five populations identified in NGC 2808. In Section 4 we discuss the interpretation of the observational findings. We summarize our conclusions in Section 5.

\section{DATA SETS AND REDUCTION}

We have compiled high-precision PM catalogs for the central regions of $22 \mathrm{GCs}$, mostly based on the archival HST proposal AR-12845 (P.I.: Bellini, see Bellini et al. 2014). PMs are obtained following the central overlap method (Eichhorn \& Jefferys 1971) via a careful data-rejection process. Extensive simulations have demonstrated the reliability of the estimated PMs and their errors. The observations used to compute PMs in NGC 2808 are listed in Table 11 of Bellini et al. (2014). We additionally included WFC3/UVIS observations of GO-12605 $(6 \times 650 \mathrm{~s}$ in F336W and $6 \times 97 \mathrm{~s}$ in F438W), taken in 2013, to further extend the available time baseline and increase the PM accuracy. The catalog contains over 86000 stars down to $\sim 5$ mag below the turn off (TO). Well-measured stars have a

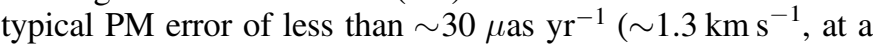
distance of $9.6 \mathrm{kpc}$, Harris 1996).

The photometric catalog of Milone et al. (2015b) combines the GO-10775 F606W and F814W ACS/WFC photometry presented in Anderson et al. (2008) with the WFC3/UVIS F275W, F336W and F438W exposures of GO-12605. All observations were corrected for CTE effects (Anderson \& Bedin 2010) and reduced using the software family img $2 x y m$ (Anderson \& King 2006), employing spatially varying, timedependent empirical PSFs (Bellini et al. 2013a). Stellar positions were corrected for geometric distortion following the recipes in Bellini \& Bedin (2009) and Bellini et al. (2011). Photometry is calibrated as in Bedin et al. (2005).
We refer to Bellini et al. (2014) and Milone et al. (2015b) for a detailed description of the data reduction.

The cross-identification of common stars between the PM and the photometric catalog left us with nearly 38000 objects down to about 3 mag below the TO. In order to analyze in detail the internal kinematics of NGC 2808 stars, particular care must be taken in removing any PM measurements affected by systematic effects. To this aim, we closely followed the recipes described in detail in Section 7.5 of Bellini et al. (2014). Our final sample consists of about 27000 stars within 115" (the outermost corner of the field of view) with high-precision, high-quality measurements.

\section{THE INTERNAL KINEMATICS OF THE FIVE SUBPOPULATIONS}

To avoid edge effects, we consider hereafter only stars within $2 \times r_{\mathrm{h}}=96^{\prime \prime}$ (Harris 1996, 2010 edition). Due to small-number statistics, the kinematics of the MSPs on the RGB cannot be studied with sufficient detail. On the other hand, we have plenty of MS stars at our disposal, in particular: 598 population-A stars, $3071 \mathrm{~B}, 1875 \mathrm{C}, 4485 \mathrm{D}$, and $1814 \mathrm{E}$. We will use the same nomenclature and color-coding of Milone et al. (2015b).

The 1D average transverse velocity-dispersion profile $\sigma_{\mu}$ of each population, obtained by combining together $\mu_{\alpha} \cos \delta$ and $\mu_{\delta}$, is shown in panel (a) of Figure 1. We binned each population in such a way as to have the same number of measurements $(\gtrsim 150)$ in each bin. We marked the position of $r_{\mathrm{h}}$ (inner dashed line), $1.5 \times r_{\mathrm{h}}$ (dotted line), and $2 \times r_{\mathrm{h}}$ (outer dashed line). All error bars refer to $1 \sigma$ errors.

The five profiles largely follow the same trend. To better examine small velocity-dispersion differences among them we proceeded as follows. We chose population B (He-normal, 1G stars) as a reference, and we least-squares fitted a 3rd-order polynomial to its $\sigma_{\mu}$ profile (red points in panel a) to obtain the average trend $\left\langle\sigma_{\mu}^{\mathrm{B}}\right\rangle$. Then, we computed the differences $\Delta \sigma_{\mu}^{\mathrm{X}}$ between the $\sigma_{\mu}$ of the other four populations $(\mathrm{X}=\mathrm{A}-\mathrm{E})$ and the average trend of population $\mathrm{B}$ at the same radial distance. These quantities are then normalized to the average trend of population B itself. These normalized differences are shown in panels (b)-(e), as a function of $r$, for populations A, C, D, and $\mathrm{E}$, respectively.

In addition, we binned each population into three radial intervals: (1) $r \leqslant r_{\mathrm{h}}, \quad$ (2) $r_{\mathrm{h}}<r \leqslant 1.5 \times r_{\mathrm{h}}$, and (3) $1.5 \times r_{\mathrm{h}}<r \leqslant 2 \times r_{\mathrm{h}}$. The respective values of $\Delta \sigma_{\mu}^{\mathrm{X}} /\left\langle\sigma_{\mu}^{\mathrm{B}}\right\rangle$ for these radial bins are shown as black points with errorbars. Populations A and C (panels (b) and (c)) exhibit slightly increasing $\Delta \sigma_{\mu}^{\mathrm{X}} /\left\langle\sigma_{\mu}^{\mathrm{B}}\right\rangle$ values the larger the radial distance, while populations D and E (panels (d) and (e)) seem to have the opposite behavior. These differences are marginally significant (at the $2 \sigma$ level).

We repeated the same analysis shown in Figure 1 independently for the radial and tangential components of motion. ${ }^{10}$ Results are shown in Figures 2 and 3 for the radial $\left(\sigma_{\text {rad }}\right)$ and tangential $\left(\sigma_{\tan }\right)$ velocity-dispersion profiles, respectively.

First, we note the overall trend of $\sigma_{\tan }$ (Figure 3(a)) being smaller than $\sigma_{\text {rad }}$ (Figure 2(a)) for $r \gtrsim r_{\mathrm{h}}$, in agreement with what found by Watkins et al. (2015) for the RGB of this cluster. The radial velocity dispersions of the five populations do not

\footnotetext{
${ }^{10}$ We decreased the number of bins while keeping the same number of measurements within (at least 150) for populations A, D, and E.
} 

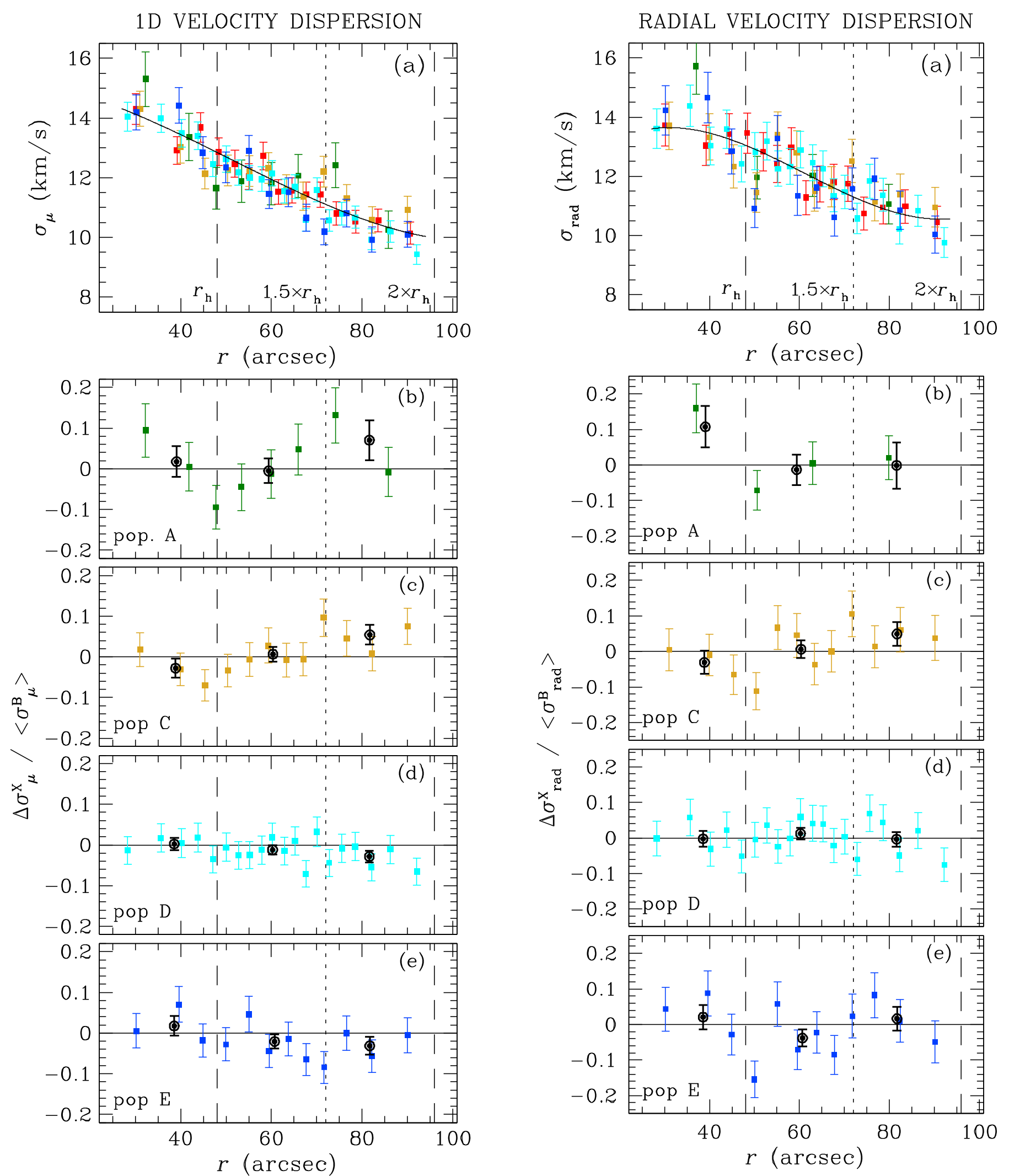

Figure 1. (a) 1D $\sigma_{\mu}$ vs. $r$ for the five distinct populations. We least-squares fitted a 3rd-order polynomial to the population B profile $\left(\left\langle\sigma_{\mu}^{\mathrm{B}}\right\rangle\right)$ (black curve). The half-light radius, $1.5 \times r_{\mathrm{h}}$, and $2 \times r_{\mathrm{h}}$ (dashed and dotted lines) are also indicated. Panels (b)-(e) show the normalized difference between $\sigma_{\mu}$ of populations $\mathrm{A}, \mathrm{C}, \mathrm{D}$, and $\mathrm{E}$, respectively, with respect to that of the reference population B. Colored points refer to bins containing the same number of stars per population. Black points refer to three fixed radial intervals: (1) $r \leqslant r_{\mathrm{h}}$, (2) $r_{\mathrm{h}}<r \leqslant 1.5 \times r_{\mathrm{h}}$, and (3) $1.5 \times r_{\mathrm{h}}<r \leqslant 2 \times r_{\mathrm{h}}$. See the text for details.

Figure 2. Similar to Figure 1 except for the radial component of the motion. See the text for details.

show significant differences (with only possible hints, at less than $\sim 2-\sigma$ level, of a larger $\sigma_{\text {rad }}$ of population $\mathrm{A}$ in the innermost region, a larger $\sigma_{\text {rad }}$ for population $\mathrm{C}$ in the outermost bin), while for $r>1.5 \times r_{\mathrm{h}}$ the tangential velocity 
TANGENTIAL VELOCITY DISPERSION
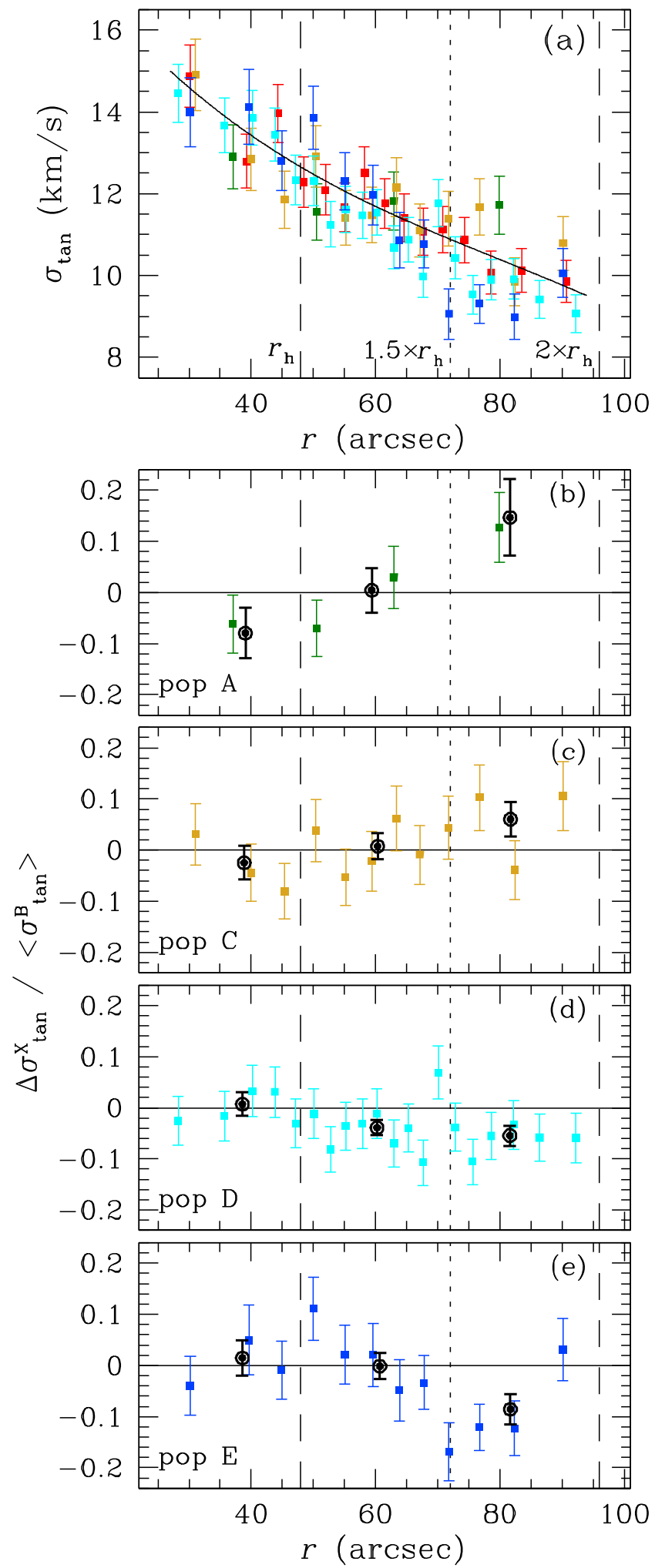

Figure 3. Similar to Figure 1 except for the tangential component of the motion. See the text for details.

dispersion of populations D and E (panels (d) and (e) of Figure 3$)$ is significantly smaller $(-0.054 \pm 0.019$ for $\mathrm{D}$, and $-0.086 \pm 0.028$ for $\mathrm{E}$, i.e., at the 2.8 and $3.1 \sigma$ levels, respectively) than that of the reference population $B$. No

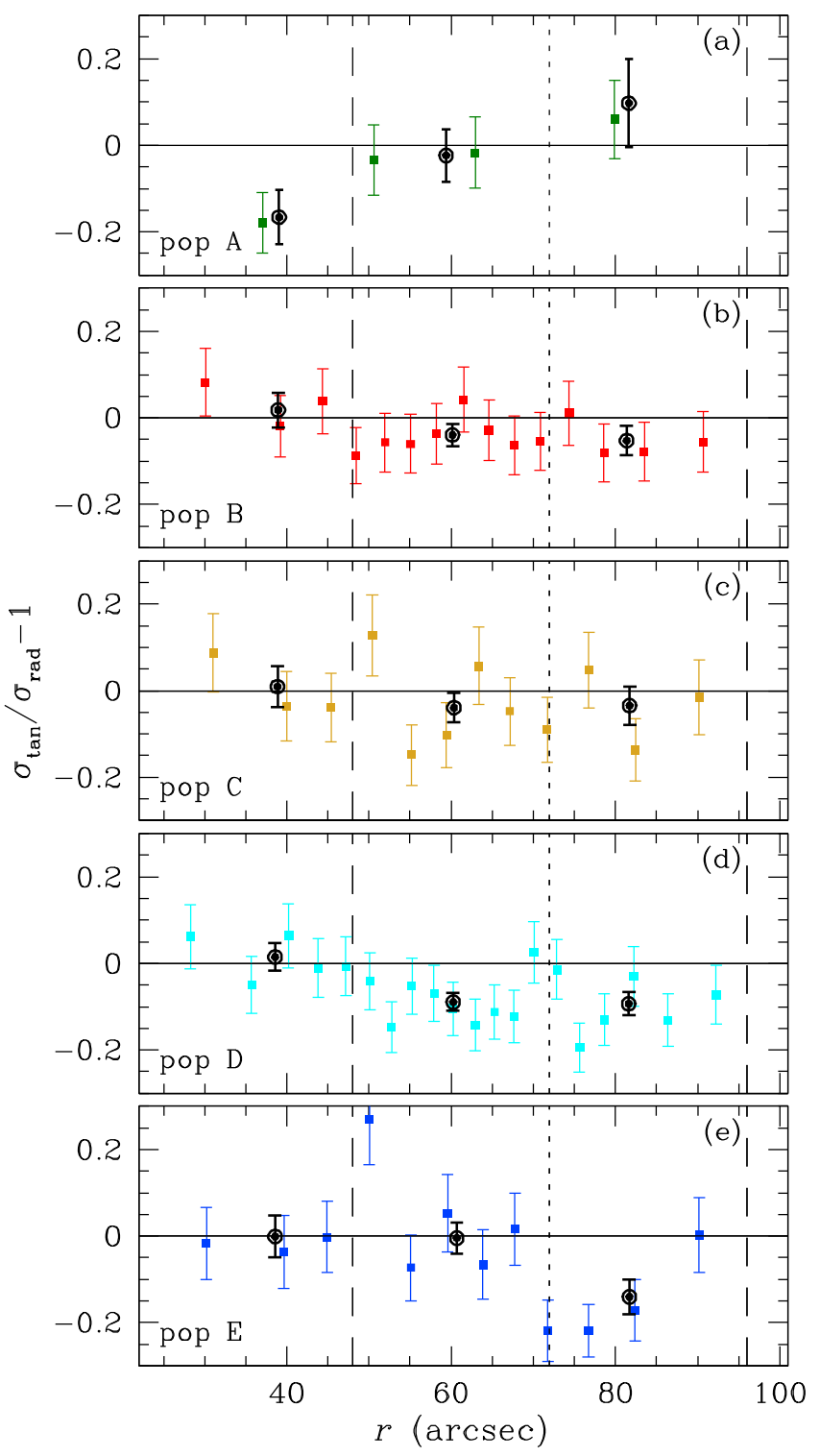

Figure 4. Deviation from tangential-to-radial isotropy (horizontal line) for the five populations (color-coded as in Figure 1). Vertical lines mark the locations of $r_{\mathrm{h}}, 1.5 \times r_{\mathrm{h}}$, and $2 \times r_{\mathrm{h}}$.

significant difference is instead found between the kinematics of population $\mathrm{B}$ and that of $\mathrm{A}$ and $\mathrm{C}$, with only a hint (at less than $\sim 2 \sigma$ level) of a larger tangential velocity for populations $\mathrm{A}$ and $\mathrm{C}$ in the outermost regions (panels (b) and (c) of Figure 3).

To further explore these findings, we computed the radial dependence of the deviation from isotropy $\left(\sigma_{\tan } / \sigma_{\text {rad }}-1\right)$ of each population. Results are shown in Figure 4. The horizontal line at 0 corresponds to an isotropic system. As for Figure 1, larger black dots refer to data binned in the three radial intervals, while colored points are used for data binned by keeping the same number of stars per bin.

Populations $\mathrm{B}$ and $\mathrm{C}$ have an overall similar trend, and tend to deviate very slightly from isotropy outside $r_{\mathrm{h}}$. In the outermost regions probed (between $1.5 r_{\mathrm{h}}$, and $2 r_{\mathrm{h}}$ ), populations $\mathrm{D}$ and $\mathrm{E}$ are radially anisotropic $\left(\sigma_{\mathrm{tan}} / \sigma_{\mathrm{rad}}-1\right.$ equal to $-0.093 \pm 0.027$ for $\mathrm{D}$ and $-0.140 \pm 0.040$ for $\mathrm{E}:$ a deviation from an isotropic distribution at the $\sim 3.4 \sigma$ and $3.5 \sigma$ levels, 
respectively). There is a hint of population A having the opposite behavior with respect to the others: the outer two points are fairly consistent with an isotropic system, while the innermost point indicates a marginally significant (at the $\sim 2 \sigma$ level) radial anisotropy for $r<r_{\mathrm{h}}$. As discussed in the Introduction, the nature of population $\mathrm{A}$ is still unclear and spectroscopic data will be necessary to characterize this population in the context of the MSPs of NGC 2808. Additional kinematic data would also be necessary to shed further light on the kinematic properties of this population and clarify the significance of possible kinematic anomalies.

\section{THEORETICAL INTERPRETATION}

The two most statistically significant results presented in the previous sections are: (i) populations $\mathrm{D}$ and $\mathrm{E}$ have a radially anisotropic velocity distribution (Figure 4) in the outermost regions, and (ii) this difference in anisotropy is due to the fact that populations $\mathrm{D}$ and $\mathrm{E}$ have a smaller tangential velocity dispersion than that of the other populations (Figure 3). No strong differences are instead found in the radial velocity dispersion of all the populations (see Figure 2).

One of the predictions of MSP cluster-formation models, in which asymptotic-giant branch (AGB) stars are the source of gas out of which $2 \mathrm{G}$ stars form, is that the AGB ejecta would collect in the cluster central regions and $2 \mathrm{G}$ stars would initially be more spatially concentrated than the $1 \mathrm{G}$ population (D'Ercole et al. 2008). Other formation models, based on rapidly rotating massive stars and/or massive binaries (see, e.g., Decressin et al. 2007; de Mink et al. 2009; Bastian et al. 2013) do not follow the evolution of the processed gas, but assume that massive stars are initially concentrated in the cluster inner regions and so is the gas they release. In any case, the subsequent long-term dynamical evolution will gradually erase the differences in the spatial distribution of $1 \mathrm{G}$ and $2 \mathrm{G}$ stars.

Here, we present the results of an $N$-body simulation carried out to explore the evolution of the kinematical properties of different stellar populations. We started our simulation with 50000 equal-mass particles, with $1 \mathrm{G}$ and $2 \mathrm{G}$ populations having the same total mass and both following a King (1966) model density profile with central dimensionless potential $W_{0}=7$. We assumed that the $2 \mathrm{G}$ population is more centrally concentrated, with an initial half-mass radius about 4.5 times smaller than that of the $1 \mathrm{G}$ population. As for the kinematical properties, both $1 \mathrm{G}$ and $2 \mathrm{G}$ systems are initially isotropic. The cluster is initially tidally limited and is assumed to move on a circular orbit in the external potential of the host galaxy (modeled as a point mass). We point out that we are not ruling out the possibility that the formation process and the subsequent virialization phase might result in $1 \mathrm{G}$ and $2 \mathrm{G}$ subsystems with initially different anisotropy profiles, but here we focus solely on the role of relaxation-driven long-term dynamical evolution in establishing kinematical differences between different stellar populations. This simulation has been run with the GPU-accelerated version of the NBODY6 code (Aarseth 2003; Nitadori \& Aarseth 2012) on the Big Red II supercomputer at Indiana University.

Observational data reveal that the strongest deviation from isotropy occurs in the outermost regions for which data are available. Figure 5 shows the time evolution of $\sigma_{\tan } / \sigma_{\text {rad }}-1$ measured for particles between 1.5 and $2 r_{\mathrm{h}}$ (where $r_{\mathrm{h}}$ is the projected half-mass radius) for the $2 \mathrm{G}$ and the $1 \mathrm{G}$ populations,

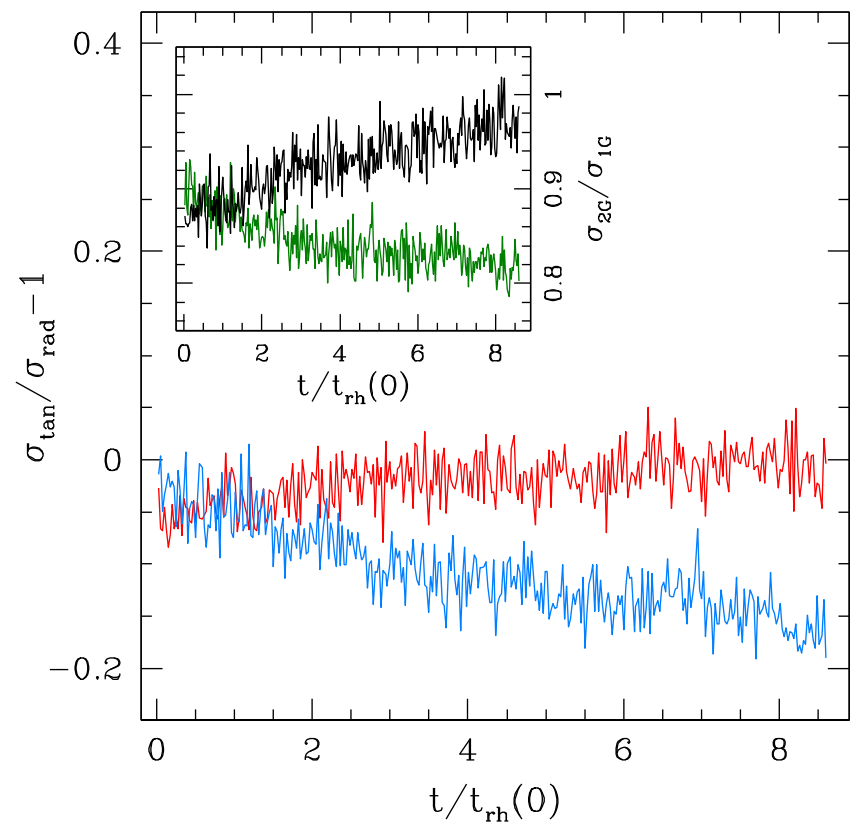

Figure 5. Time evolution of $\sigma_{\tan } / \sigma_{\text {rad }}-1$ measured for particles between 1.5 and $2 r_{\mathrm{h}}$ for the $2 \mathrm{G}$ (blue line) and the $1 \mathrm{G}$ (red line) populations (where $r_{\mathrm{h}}$ is the projected half-mass radius). The green line in the inset shows the time evolution of the ratio of the $2 \mathrm{G}$ to the $1 \mathrm{G}$ tangential velocity dispersion, while the black line is for the ratio of the $2 \mathrm{G}$ to the $1 \mathrm{G}$ radial velocity dispersion (both measured between 1.5 and $2 r_{\mathrm{h}}$ ). Time is normalized to the initial half-mass relaxation time (calculated using the projected half-mass radius).

where $\sigma_{\text {rad }}$ and $\sigma_{\tan }$ are, respectively, the projected radial and tangential velocity dispersions as measured on a plane coinciding with the cluster orbital plane. This is the outermost region probed by our observational data where the difference in anisotropy between $1 \mathrm{G}$ and $2 \mathrm{G}$ populations is strongest. The results of our simulation show a trend qualitatively consistent with that found in the observational data: as $2 \mathrm{G}$ stars diffuse from the inner regions (where they formed and are initially segregated), they populate the outer cluster regions preferentially on radial orbits as shown by the increasing radial anisotropy of the $2 \mathrm{G}$ population. The trend found in our simulation agrees with what was found in a recent numerical study by Henault-Brunet et al. (2015). The different formation and dynamical history of $1 \mathrm{G}$ and $2 \mathrm{G}$ stars, which are currently located in the same outer region of the cluster (in this case between 1.5 and $2 r_{\mathrm{h}}$ ), is revealed by the differences in the kinematic properties. These differences are stronger in the cluster's outer regions, which are initially dominated by $1 \mathrm{G}$ stars. As the cluster evolves, the cluster's outer regions are populated also by $2 \mathrm{G}$ stars, diffused from their initial inner location.

The inset of Figure 5 shows the time evolution of the ratio of the $1 \mathrm{G}$ to the $2 \mathrm{G} \sigma_{\text {rad }}$ and $\sigma_{\tan }$, measured between 1.5 and $2 r_{\mathrm{h}}$. The initial differences in the $\sigma_{\text {rad }}$ and $\sigma_{\tan }$ of the two populations evolve in a direction consistent with the observed trends: the radial velocity dispersion $\sigma_{\text {rad }}$ of the two populations becomes increasingly similar while $\sigma_{\tan }$ of the $2 \mathrm{G}$ becomes smaller than that of the $1 \mathrm{G}$ and, in agreement with our observational findings. It is this difference that is responsible for the differences in the anisotropy of the two populations. 


\section{CONCLUSIONS}

In this Letter we have presented an $H S T$ study of the kinematical properties of the MSPs of NGC 2808 based on high-precision PM measurements (Bellini et al. 2014). In a recent study, Milone et al. (2015b) identified five different populations in this cluster: two of these populations (named D and $\mathrm{E}$ ) correspond to the $2 \mathrm{G}$ populations in the $\mathrm{mMS}$ and $\mathrm{bMS}$ identified in an earlier study by Piotto et al. (2007) while the other three populations (A-C) correspond to the rMS population in the Piotto et al.'s study. In this Letter we show that the five stellar populations of this cluster are characterized by differences in their kinematics.

Specifically, we find that in the outermost regions probed (between 1.5 and $2 r_{\mathrm{h}}$ ) the velocity distribution of the $2 \mathrm{G}$ populations $\mathrm{D}$ and $\mathrm{E}$ is radially anisotropic (the deviation from isotropy being significant at the $\sim 3.5 \sigma$ level). Our data show that the larger radial anisotropy in the $2 \mathrm{G}$ populations $\mathrm{D}$ and $\mathrm{E}$ is due to the fact that these populations have smaller tangential velocity dispersions than the other populations. Qualitatively similar trends have been found by Richer et al. (2013) in 47 Tuc. No significant differences are found in the radial velocity dispersions of all the populations and between the kinematics of population $\mathrm{B}$ and that of $\mathrm{A}$ and $\mathrm{C}$ (with only possible hints of a larger $\sigma_{\text {rad }}$ of population $\mathrm{A}$ in the innermost region, a larger $\sigma_{\text {rad }}$ for population C in the outermost bin, and a larger $\sigma_{\tan }$ for populations $\mathrm{A}$ and $\mathrm{C}$ in the outermost regions all at less than $2 \sigma$ level).

The results of an $N$-body simulation show that the differences in both the radial anisotropy and in the tangential velocity dispersion observed for populations $\mathrm{D}$ and $\mathrm{E}$ are consistent with being the kinematical fingerprint of the diffusion of $2 \mathrm{G}$ populations from the innermost regions, where they are initially concentrated, toward the cluster outer regions. A more extended survey of models, probing broader ranges of different initial conditions, is necessary to fully explore the possible evolutionary paths of the $1 \mathrm{G}$ and $2 \mathrm{G}$ kinematical properties and their dependence on initial conditions.

On the observational side, we emphasize the importance of extending the current study to even more external cluster regions. In fact, the outermost regions are essential for a complete characterization of a cluster's kinematical properties and, in particular, to shed light on the possible effects of the external Galactic tidal field. These effects are expected to limit the outer development of the radial anisotropy and make these more isotropic (and possibly even slightly tangentially anisotropic).

We plan to extend the analysis presented here to other GCs as possible, using the PM catalogs of Bellini et al. (2014) in combination with the UV photometry of Piotto et al. (2015).
This will create a substantial case history that will help us in better understanding formation and evolution of MSPs in GCs.

Support for this work comes from STScI grants for HST programs AR-12845 and GO-13297. E.V. acknowledges support by grant NASA-NNX13AF45G. G.P., S.C., F.D'A. and A.R. acknowledge support from PRIN-INAF 2014 (PI: S. Cassisi).

\section{REFERENCES}

Aarseth, S. J. 2003, Gravitational N-Body Simulations (Cambridge: Cambridge Univ. Press)

Anderson, J., \& Bedin, L. R. 2010, PASP, 122, 1035

Anderson, J., \& King, I. R. 2006, ACS/ISR 2006-01 (Baltimore, MD: STScI), available online at http://www.stsci.edu/hst/acs/documents/isrs

Anderson, J., Sarajedini, A., Bedin, L. R., et al. 2008, AJ, 135, 2055

Anderson, J., \& van der Marel, R. P. 2010, ApJ, 710, 1032

Bastian, N., Lamers, H. J. G. L. M., de Mink, S. E., et al. 2013, MNRAS, 436, 2398

Bedin, L. R., Cassisi, S., Castelli, F., et al. 2005, MNRAS, 357, 1038

Bellini, A., Anderson, J., \& Bedin, L. R. 2011, PASP, 123, 622

Bellini, A., Anderson, J., Salaris, M., et al. 2013a, ApJL, 769, L32

Bellini, A., Anderson, J., van der Marel, R. P., et al. 2014, ApJ, 797, 115

Bellini, A., \& Bedin, L. R. 2009, PASP, 121, 1419

Bellini, A., Piotto, G., Bedin, L. R., et al. 2009a, A\&A, 507, 1393

Bellini, A., Piotto, G., Bedin, L. R., et al. 2009b, A\&A, 493, 959

Bellini, A., Piotto, G., Milone, A. P., et al. 2013b, ApJ, 765, 32

Cordero, M. J., Pilachowski, C. A., Johnson, C. I., et al. 2014, ApJ, 780, 94

D’Antona, F., Bellazzini, M., Caloi, V., et al. 2005, ApJ, 631, 868

D’Ercole, A., Vesperini, E., D’Antona, F., McMillan, S. L. W., \& Recchi, S. 2008, MNRAS, 391, 825

de Mink, S. E., Pols, O. R., Langer, N., \& Izzard, R. G. 2009, A\&A, 507, L1

Decressin, T., Meynet, G., Charbonnel, C., Prantzos, N., \& Ekstrom, S. 2007, A\&A, 464, 1029

Eichhorn, H., \& Jefferys, W. H. 1971, PMcCO, 16, 267

Gratton, R. G., Carretta, E., \& Bragaglia, A. 2012, A\&ARv, 20, 50

Harris, W. E. 1996, AJ, 112, 1487 (2010 edition)

Henault-Brunet, V., Gieles, M., Agertz, O., \& Read, J. I. 2015, MNRAS, 450,1164

Johnson, C. I., \& Pilachowski, C. A. 2012, ApJL, 754, L38

King, I. R. 1966, AJ, 71, 64

Lardo, C., Bellazzini, M., Pancino, E., et al. 2011, A\&A, 525, A114

Marino, A. F., Milone, A. P., Przybilla, N., et al. 2014, MNRAS, 437, 1609

Milone, A. P., Marino, A. F., Piotto, G., et al. 2015a, MNRAS, 447, 927

Milone, A. P., Marino, A. F., Piotto, G., et al. 2015b, ApJ, 808, 51

Milone, A. P., Piotto, G., Bedin, L. R., et al. 2012, A\&A, 537, AA77

Nardiello, D., Piotto, G., Milone, A. P., et al. 2015, arXiv:1504.07876

Nitadori, K., \& Aarseth, S. J. 2012, MNRAS, 424, 545

Pasquini, L., Mauas, P., Käufl, H. U., \& Cacciari, C. 2011, A\&A, 531, A35

Piotto, G., Bedin, L. R., Anderson, J., et al. 2007, ApJL, 661, L53

Piotto, G., Milone, A. P., Bedin, L. R., et al. 2015, AJ, 149, 91

Richer, H. B., Heyl, J., Anderson, J., et al. 2013, ApJL, 771, L15

Sollima, A., Ferraro, F. R., Bellazzini, M., et al. 2007, ApJ, 654, 915

Vesperini, E., McMillan, S. L. W., D’Antona, F., \& D’Ercole, A. 2013, MNRAS, 429, 1913

Villanova, S., Geisler, D., Piotto, G., \& Gratton, R. G. 2012, ApJ, 748, 62

Watkins, L. L., van der Marel, R. P., Bellini, A., \& Anderson, J. 2015, ApJ, 803,29 